UDC 37:81'373.7

DOI https://doi.org/10.32838/2663-6069/2020.2-2/40

Svyrydjuk $V . P$.

Nationale linguistische Universität

\title{
AUSTRIAZISMEN IM DEUTSCHUNTERRICHT FÜR DIE ENTWICKLUNG DER SPRACHKOMPETENZ BEI DEN STUDENTEN-PHILOLOGEN
}

The article deals with the development of sociocultural competence through the use of Austrianism in German language classes, and also focuses on the pluricentricity of German as a prerequisite for the formation of linguistic and cultural competence in foreign language communication in the German language. Particular attention is focused on the complex of exercises for acquaintance with the Austrian version of German at the lexical level.

The article draws attention to the formation of phonetic skills and abilities in order to promote and interchange Austracisms in German classes.

This article presents some examples of exercises for learning to use different online-exercises the Austrian words. The benefits of different methods of using the Austrian words the content of German lesson based on the estimated-activity methodology.

The paper presents the results of the National Linguistic University during self-education of students of bachelors. The author analyzed modern strategies of teaching new words in the communicative situations.

The article deals with the sociolinguistic component literally affects all linguistic communication between representatives of different cultures, although the participants may not be aware of this influence.

It is presented those linguistic features of Austrian German that are codified as standard German in the dictionaries.

The article is devoted to finding ways to optimize the development of writing skills students philologists. The description of interactive online exercises for application in the German language spontaneous language training is provided. The article uncovers examples of interactive exercises for teaching formulations of sentences in German language.

Key words: methods, development of educational material, national version of Austrian German, sociolinguistic competence, phonetic competence, lexical competence.

Für die Entwicklung der kommunikativen Kompetenz in der deutschen Sprache orientieren sich die Lehrenden an den Lernzielen, die im Gemeinsamen Europäischen Referenzrahmen festgelegt und im Curriculum für DaF-Lernende und -Lehrende beschrieben worden sind $[1 ; 12]$. Im Rahmen des Gesamtlernziels kommunikativer Kompetenz werden die Fähigkeiten hervorgehoben, die die nationalen Brücken zwischen den deutschsprachigen Ländern bilden. In diesem Zusammenhang soll nach der Meinung vieler europäischer Didaktiker die deutsche Sprache nicht nur monozentristisch, sondern vielmehr plurizentristisch verstanden werden $[2 ; 4 ; 6 ; 9]$.

Die Analyse einiger Lehrwerke $[1 ; 2 ; 3]$, die den Deutschlernenden zur Verfügung stehen, zeigt, dass die plurizentrischen Merkmale der deutschen Sprache implizit vermittelt werden. Das betrifft in erster Linie die Hörfertigkeiten, wenn die Lernenden das Gehörte wahrnehmen und verstehen müssen. Nach den Anfor- derungen der oben genannten Dokumente sollen die DaF-Lernenden schon auf dem Niveau B2 globales Verstehen von nationalen Standardvarietäten beherrschen. So sollen sie auf diesem Niveau österreichische und Schweizer Varianten des Deutschen üben, damit dann auf dem Niveau C1 das vollständige Verstehen erreicht werden kann $[1 ; 12]$.

Im Gemeinsamen Europäischen Referenzrahmen für Sprachen ist unter dem Punkt soziokulturelles Wissen aufgeführt, dass ab B1-Niveau passive Kenntnisse über die nationalen Varietäten des Deutschen verlangt werden dürfen und im weiteren Prozess des Deutschlernens sollen die DaF-Lernenden und -Lehrenden auf C1-Niveau soziolinguistische Unterschiede der nationalen Varietäten identifizieren [12, S.104]. Soziolinguistische Kompetenzen sind definiert durch die soziokulturellen Bedingungen der Sprachverwendung. Die soziolinguistische Komponente wirkt buchstäblich auf alle sprachliche Kom- 
munikation zwischen Repräsentanten verschiedener Kulturen, obgleich die Gesprächsteilnehmer sich dieses Einflusses oft gar nicht bewusst sein mögen [12, S. 25].

Die Deutschlernenden an den linguistischen Fakultäten sollen sich hinsichtlich des Curriculums auf dem B1-Niveau mit den Merkmalen der nationalen Varietäten auseinandersetzen, während auf dem C1-Niveau alle Gesprächspartner/-innen aus dem ganzen deutschsprachigen Raum verstanden werden müssen [1, S. 105].

Ausserdem sollten die DaF-Lernenden die nationalen Varietäten selber kennen lernen, weil sie oft in einem deutschsprachigen Land weiterstudieren oder beruflich tätig wollen. Im Unterricht sollen deshalb Kenntnisse zu den Varietäten aufgebaut werden.

Als Einstieg in die Plurizentrik sollen zumindest passive Kenntnisse darüber vorhanden und einige Grundbegriffe geklärt sein. Die Untersuchungen der Didaktiker in diesem Bereich zeigen, dass das Erlernen der nationalen Varietäten eine spezielle Vorbereitung braucht, weil sie auf der Basis des Standarddeutschen beherrscht werden sollen.

In der ersten Phase der Bekanntschaft mit der plurizentrischen Sprache erfahren die Lernenden, dass drei interagierende Zentren (Deutschland, Österreich und Schweiz) jeweils über eigene nationale Standardvarietät verfügen, die eigene kodifizierte Normen aufweisen $[2 ; 8 ; 9]$. Wenn die DaF-Lernenden in den Varietäten aus einem der deutschsprachigen Ländern kommunizieren, sollen sie soziolinguistische Merkmale des Deutschen wahrnehmen und verstehen, um bei der Kommunikation erfolgreich zu sein.

Es gibt drei wesentliche Gründe für die Vermittlung von Standardvarietäten im DaF-Unterricht:

1) Bedarfserhebung durch offene Grenze zwischen den Ländern;

2) die Realität der gesprochenen Sprache miterleben;

3) Vorgaben des Europäischen Referenzrahmens [12].

Auf diese Weise sollen die nationalen Varietäten im DaF-Unterricht berücksichtigt und zielbewusst vermittelt werden.

In diesem Beitrag wird anhand aktueller Forschungsergebnisse und durch praktische Erfahrung die nationale Varietät des österreichischen Deutschen im Bereich rezeptiver Aussprachefertigkeiten im DaF-Unterricht thematisiert.

Die von R. de Chillia, P. Wiesinger, K.Reinke und anderen Didaktikern diskutierte Frage der österreichischen Varietäten im DaF-Unterricht ermuntert zum Heraussuchen der neuen Methoden für die Entwick- lung der Lern- und Lehrmaterialien $[9 ; 10 ; 14]$ In diesem Fall sollen von den Lehrenden die DACH-Prinzipien eingehalten werden, damit die Lernenden sich bewusst sind, dass die deutsche Sprache in jedem deutschsprachigen Land ihre linguistische Besonderheiten hat. Dazu kommen folgende Prinzipien:

1) das rezeptionsorientierende Prinzip;

2) das lernorientierende Prinzip und

3) das prozessorientierende Prinzip [3; 9; 10;13]

Dabei soll betont werden, dass wir diejenigen linguistischen Besonderheiten des österreichischen Deutsch anbieten, die in den Wörterbüchern als Standarddeutsch kodifiziert sind $[2 ; 4 ; 8]$. Wir unterstützen die Meinungen von U.Hirschfeld, R.Muhr K.Reinke, dass im DaF-Unterricht immer die Vielfalt des deutschsprachigen Raums anzuerkennen ist und die Zielsprache Deutsch auf mehr als ein Land verteilt ist $[6 ; 8 ; 9]$. Wir sind überzeugt, dass sich im Sprachunterricht gleichzeitig Landeskunde anhand der deutschsprachigen Lernmaterialien vermitteln lässt. Den Lernenden stehen beim Hören und Sprechen nationale Varianten z.B. des österreichischen Deutsch zur Verfügung, damit sie sie aktiv und rezeptiv benutzen können. Das Lernziel der praktischen Übung ist, dass die Lernenden im gesamtdeutschsprachigen Raum möglichst viel verstehen und mit linguistischen Unterschieden und Gesamtheiten gut umzugehen lernen. Hinsichtlich der heutigen Anforderungen auf dem Arbeitsmarkt und moderner Innovationen sollte sich der DaF-Unterricht der sprachlichen Realität annäheren.

Man muss sagen, dass die Plurizentrik des Deutschen in den ukrainischen Lehrwerken kaum Berücksichtigung finden. Die Thematik des plurizentrischen Deutsch wird implizit eingebettet $[1 ; 2 ; 3]$.

Unsere Untersuchungen zeigen, dass die Lernmaterialien, die die nationalen Merkmale des österreichischen Deutsch enthalten, von den Lehrenden oft nicht erklärt werden und damit erzeugt die Angelegenheit bei den Lernenden keine Aufmerksamkeit. Ausserdem gibt es beim Modellieren der Gesprächssituationen kein entsprechendes Land, in welchem die nationalen Merkmale des Deutschen auftauchen können. Überwiegend wird im Unterricht eine Gesprächssituation im allgemeinen deutschsprachigen Raum modelliert, die in erster Linie Deutschland repräsentiert. Man soll demnach öfter berücksichtigen, dass österreichisches oder Schweizer(hoch)deutsch also genauso korrekt und akzeptabel wie das Standarddeutsche der Bundesrepublik Deutschland $[7 ; 8 ; 11]$.

Im Laufe des Deutscherlernens sollte der Reichtum an Vielfalt der Kultur und Sprache den Lernenden vermittelt werden. Dadurch werden die linguistischen 
Spezifika z.B. von Österreich präsentiert. Die Lernenden können damit im alltäglichen Leben leichter mit den Muttersprachlern ins Gespräch kommen und sich im Land sicherer fühlen.

Wir bieten einige Übungen und Aufgaben hinsichtlich der praktischen Phonetik an, weil dieser Aspekt als wichtiger Bestandteil der Kommunikationskultur eine wesentliche Rolle spielt. Von den Fähigkeiten des Wahrnehmens und des Verstehens hängt ja die gelingende Kommunikation ab.

Die von U.Ammon beschriebenen linguistischen Merkmale des österreichischen Deutsch sind in den Beiträgen von R.Muhr, P.Wiesinger und B.Kellermeier-Rehbein weiter auf den grammatischen, lexikalischen und phonetischen Ebenen konkretisiert $[2 ; 7 ; 8 ; 14]$.

Da im DaF-Unterricht überwiegend viele lexikalische Einheiten gelernt werden sollen, beachten wir phonologische Prozesse, die sich auf die Aussprache einiger Laute im österreichischen Deutsch beziehen.

Beim Erarbeiten von solchem phonetischen Lernmaterial steht nicht nur die Entwicklung rezeptiver Fähigkeiten, sondern auch die Vermittlung des Wissens zur Verfügung. Hierfür genügt es nicht, sich z.B. auf die Inhalte des Hörverstehens zu konzentrieren, man muss ebenso die Lern- und Wissenskompetenz berücksichtigen. Dabei sollen die Deutschlernenden unseres Erachtens zu folgenden Punkten etwas wissen und lernen:

1) lexikalische Einheiten, dazu gehören auch Austriazismen in verschiedenen Bereichen des alltäglichen Lebens;

2) Aussprachebesonderheiten der Vokale und Konsonanten im österreichischen Deutsch;

3) soziokulturelles Verhalten beim Sprechen, z.B. Toleranz zeigen; Blickkontakt halten.

Es steht ausser Zweifel, dass der Aufbau einer Wahrnehmungstoleranz gegenüber dem Deutschen in jedem Fall mit der Standardaussprache beginnen sollte.

Eine Reihe der Übungen und Aufgaben, die in diesem Beitrag angeboten werden, hat das Ziel, auf der linguistischen Ebene einen Einstieg in den plurizentrischen Ansatz für die deutsche Sprache zu schaffen und die sprachliche Realität in Österreich zu präsentieren.

Auf sprachlicher Ebene stehen die Erweiterung und das Üben des Lese- und Hörverstehens und des mündlichen Gebrauchsstandards der österreichischen Varietät im Zentrum. Zur Entwicklung der phonetischen Fertigkeiten werden in diesem Beitrag die von K. Reinke formulierten methodischen Prinzipien eingehalten, unter denen eines die Plurizentrik der deutschen Spra- che betont: „Berücksichtigung von Angemessenheit, Intention und Wirkung-Autentität, Emotionalität und Variantenbreite“ [9]. Es wird hier neben der phonostilistischen Variation auch Bezug geschaffen zu regionalen Varianten und $\mathrm{zu}$ den Varietäten (Österreich, Schweiz, Bundesrepublik Deutschland).

Das vorbereitete Material wurde von uns auf drei Ebenen entwickelt. Die erste Ebene enthält Übungen, die Vorwissen und Wissen aktivieren. Es folgen das Kennenlernen der lexikalischen Bestandteile der österreichischen Standardvarietät und die Aussprachemerkmale derjenigen Laute, durch welche sich österreichisches Deutsch im Vergleich zum Standardaussprache des Deutschen in der BRD unterscheidet. Auf der dritten Ebene wird den Deutschlernenden globales Hören angeboten, wobei sie die Besonderheiten des österreichischen Deutsch erkennen sollen. Nach dem selektiven Hörverstehen desselben Textes kommt die vierte Ebene des Sprachgebrauchs, wobei die Lernenden eine entsprechende Gesprächssituation anhand des gelernten linguistischen Materials abspielen können. Um die Übungen und Aufgaben nach den obigen Lernebenen zu entwickeln, werden folgende Übungstypen berücksichtigt: rezeptive, rezeptiv-reproduktive, reproduktive, produktive; sprachliche und kommunikative Übungen zum freien Sprechen. (s. Tabelle 1).

Wir betrachten den Lernprozess aus der Bekanntschaft mit dem österreichischen Deutsch auf der phonetischen Ebene, indem wir die Aussprache des Schwa [ə] im Suffix -el und eigentlich des [1]-Lautes im Auslaut kennen lernen. Ausserdem beobachten wir die Standardaussprache des österreichtischen Deutsch einer Ansagerin und eines Gastes durch die Präsentation eines Films im österreichischen Fernsehen (ORF).

Übung 1. Ziel: Vorwissen wecken und Wissen vermitteln

Aufgabe: Lesen Sie den Text und informieren Sie sich über die plurizentrische Sprache Deutsch. Danach absolvieren Sie einen kurzen Test zum Leseverstehen.

Beim Lesen erfahren die Lernenden, dass Deutsch eine plurizentrische Sprache ist und es unterschiedliche Standardvarietäten hat. Dazu kommen die ersten Austriazimen aus dem Text.

In der nächsten Übung erhält man einige Kenntnisse im Bereich der Plurizentrik.

Übung 2. Ziel: Wissen vermitteln

Aufgabe: Lesen Sie die folgenden Begriffe. Ordnen Sie die richtige Definition zu.

Notieren Sie den richtigen Buchstaben in der letzten Spalte. 


\begin{tabular}{|c|c|c|c|c|}
\hline 1 & die Varietät & a & $\begin{array}{c}\text { typisch österreichische } \\
\text { Ausdrücke }\end{array}$ & 1c \\
\hline 2 & der Dialekt & b & $\begin{array}{c}\text { typisch schweizerische } \\
\text { Ausdrücke }\end{array}$ & \\
\hline 3 & $\begin{array}{c}\text { das } \\
\text { Standarddeutsch }\end{array}$ & c & $\begin{array}{c}\text { Eine Sprachform } \\
\text { innerhalb einer Sprache } \\
\text { mit } \\
\text { spezifischen } \\
\text { sprachlichen } \\
\text { Eigenheiten }\end{array}$ & \\
\hline 4 & $\begin{array}{c}\text { die } \\
\text { plurizentrische } \\
\text { Sprache }\end{array}$ & d & $\begin{array}{c}\text { Die Sprache mit einer } \\
\text { lokalen regionalen } \\
\text { Färbung }\end{array}$ & \\
\hline 5 & $\begin{array}{c}\text { die } \\
\text { Umgangssprache }\end{array}$ & e & $\begin{array}{c}\text { typisch deutsche } \\
\text { Ausdrücke }\end{array}$ & \\
\hline 6 & die Austriazismen & f & $\begin{array}{c}\text { Die Sprache, die im } \\
\text { Alltag benutzt wird }\end{array}$ & \\
\hline 7 & die Teutonismen & g & $\begin{array}{c}\text { Die standardisierten } \\
\text { Sprachvarietäten im } \\
\text { deutschen Sprachraum }\end{array}$ & \\
\hline 8 & die Helvetismen & h & $\begin{array}{c}\text { eine Sprache mit } \\
\text { mehreren } \\
\text { Standardvarietäten }\end{array}$ & \\
\hline
\end{tabular}

Die Lernenden machen sich mit den Begriffen und Definitionen vertraut, welche die nationalen Varietäten der deutschsprachigen Länder klären.
Um die ersten Austriazismen zu lernen, werden den Lernenden Sätze angeboten, in denen sie die österreichischen Wörter bestimmen sollen. Beim Vorlesen der Sätze lenken wir ihre Aufmerksamkeit darauf, dass die Standardaussprache, die Soll-Norm eingehalten wird.

Übung 3. Ziel: Austriazismen im Satz erkennen.

Aufgabe: Lesen Sie die folgenden Sätze vor. Halten Sie sich an die Norm der Standardaussprache im Deutschen. Ersetzen Sie die fett gedruckten typisch österreichischen Wörter durch bundesdeutsche Wörter.

1. Die Hochzeit war sehr schön. Das Gewand der Brautmutter war sehr elegant.

2. Die Bananenschale kannst du in den Mistkübel werfen.

3. Musst du deine Hausübungen nicht machen? Du hast doch morgen Schule.

4. Ich brauche was Süsses. Hast du ein Zuckerl?

Die Aktivitäten mit den Substantiven, die dem Land Österreich zuzuordnen sind, können bereits mit dem theoretischen Material der Phonetik ergänzt werden. Man beachtet diejenigen Laute, die im österreichischen Deutsch anders ausgesprochen werden können, z.B. das R. Im österreichischen Deutsch wird

Übungsteilung für Entwicklung der soziolinguistischen Kompetenz

Tabelle 1

\begin{tabular}{|c|c|c|c|}
\hline Ebenen & Ziele des Lernmaterials & Übungen und Aufgaben & \\
\hline $\begin{array}{l}\text { 1. Einstieg } \\
\text { (z.B. } \\
\text { nationale } \\
\text { Varietät) }\end{array}$ & $\begin{array}{l}\text { Bekanntschaft mit Begriffen; } \\
\text { Vorwissen wecken }\end{array}$ & $\begin{array}{l}\text { sprachliche, rezeptive, rezeptiv- } \\
\text { reproduktive }\end{array}$ & \\
\hline $\begin{array}{l}\text { 2. Lese- } \\
\text { verstehen }\end{array}$ & $\begin{array}{l}\text { Segmentalia und suprasegmentalia der Standardaussprache } \\
\text { Deutsch beachten; } \\
\text { Austriazismen anerkennen; } \\
\text { die Aussprache des Suffixes -el; des R-Lautes; des Schwas } \\
\text { [ə], bemerken. }\end{array}$ & $\begin{array}{l}\text { sprachliche und kommunikative; } \\
\text { rezeptive }\end{array}$ & 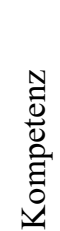 \\
\hline $\begin{array}{l}\text { 3. Hör- } \\
\text { verstehen }\end{array}$ & $\begin{array}{l}\text { Lautmerkmale [1], [ə], [R], [e] im Auslaut bei den } \\
\text { Österreichern beachten; } \\
\text { österreichische Realisierung des [1]-Lautes im Suffix -el } \\
\text { identifizieren; } \\
\text { die Aussprache des [1]-Lautes im bundesdeutschen und } \\
\text { österreichischen Deutsch; vergleichen. }\end{array}$ & $\begin{array}{l}\text { kommunikative; } \\
\text { rezeptiv-reproduktive }\end{array}$ & 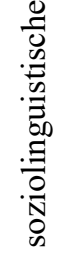 \\
\hline $\begin{array}{l}4 . \\
\text { Kommu- } \\
\text { nikation }\end{array}$ & $\begin{array}{l}\text { das phonetische Phänomen durch die erlernten segmentalen } \\
\text { Merkmale verknüpfen; } \\
\text { das phonetische Basismaterial unterscheiden; } \\
\text { sich die neuen lexikalischen Einheiten und die neuen } \\
\text { Sprachcharakteristika bewusst machen und einprägen; } \\
\text { Selbstvertrauen gewinnen; wählen und entscheiden lernen; } \\
\text { sich der österreichischen Sprachkultur nähern. }\end{array}$ & $\begin{array}{l}\text { rezeptiv-produktive; } \\
\text { kommunikative } \\
\text { produktive }\end{array}$ & \\
\hline
\end{tabular}


das $\mathrm{R}$ in der Regel als uvularer Vibrant [R] realisiert. Anlautend nach dem Plosiv [k] kommt gelegentlich auch der uvulare Frikativ [в] vor. Das Suffix -er wird das [e] sehr oft ausgesprochen.

Was die weiteren segmentalen Merkmale des österreichischen Deutsch betrifft, kann man aufgrund der Übungen sagen, dass [E], [a], [i] mit Längung ausgesprochen werden $[4$, S. 45; 8, S. 45].

Auf diese Weise kann in der nächsten Übung mit den Austriazismen die Aussprachevarietät des Deutschen vorgestellt werden. Die Deutschlernenden erklären die Austriazismen durch bundesdeutsche Wörter. Die rezepriv-reproduktive Übung hilft den Lernenden, die neuen Wörter zu lernen und ihre Aussprache mit Hilfe der Lehrkraft kennen zu lernen.

Übung 4. Ziel Austriazismen vorlesen und erschliessen.

Aufgabe: Was bedeuten folgende Austriazismen im standardsprachlichen Deutsch der BRD? Beachten Sie die Aussprachemerkmale des österreichischen Standarddeutsch. Ordnen Sie zu.

\begin{tabular}{|c|c|c|c|c|c|}
\hline 1 & der Jänner & [jहne] & $\mathrm{a}$ & der Eimer & $1 \mathrm{j}$ \\
\hline 2 & der Kübel & [kybł] & $\mathrm{b}$ & das Würstchen & \\
\hline 3 & der Fiaker & [fiake] & $\mathrm{c}$ & die Hefe & \\
\hline 4 & das Würstel & [vyвstt] & $\mathrm{d}$ & der Kutscher & \\
\hline 5 & der Germ & [gesm] & $\mathrm{e}$ & die Grundschule & \\
\hline 6 & das Zuckerl & [ts okrł] & $\mathrm{f}$ & das Abitur & \\
\hline 7 & $\begin{array}{l}\text { die } \\
\text { Volksschule }\end{array}$ & [fつlksfu:lə] & $\mathrm{g}$ & der Aufkleber & \\
\hline 8 & das Keks & [ke:ks] & $\mathrm{h}$ & das Bonbon & \\
\hline 9 & die Matura & [matu:Ra] & $\mathrm{i}$ & der Keks & \\
\hline 10 & das Pickerl & [pikeł] & $\mathrm{j}$ & der Januar & \\
\hline
\end{tabular}

Die Zielsetzung der nächsten Aufgabe ist es, die Lernenden an eine Kommunikation heranzuführen und die fremdsprachlichen Fertigkeiten zu entwickeln und zu erweitern. So wird eine Aufgabe mit den Austriazismen angeboten, damit die Lernenden die Bedeutung jedes Wortes klären können.

Übung 5. Ziel: Austriazismen kennen lernen und ihre Bedeutung erschliessen.

Aufgabe: Erklären Sie die Wörter oder notieren Sie das bundesdeutsche Wort.

\begin{tabular}{|c|c|c|}
\hline 1 & das Sackerl & \\
\hline 2 & das Kuvert & \\
\hline 3 & der Fiaker & \\
\hline 4 & der Steckerl & \\
\hline 5 & das Häferl & \\
\hline$\ldots$ & & \\
\hline
\end{tabular}

Diese rezeptiv-produktive kommunikative Aufgabe kann man sowohl mündlich als auch schrift- lich erfüllen. Beim mündlichen Erfüllen der Übung sollen die Lernenden die Realisierung des Suffuxes -erl beachten, indem es als [..eł] ausgesprochen wird [8, S. 50].

Wenn man die Aufgabe schriftlich lösen möchte, können die Austrizismen transkribiert und dann geklärt werden.

Nachdem die Lernenden die einzelnen lexikalischen Einheiten und deren Bedeutungen gelernt haben, kann man virtuell in Österreich verschiedene Dinge in der modellierten Gesprächsituation benennen.

Das Ziel der nächsten Übung ist es, die Austriazismen anhand von Bildern zu nennen. Die Bilder lassen sich beschriften und dann beschreiben. Die Lernenden haben die Möglichkeit, mit neuen Wörtern eigene Sätze zu bilden.

Übung 6. Ziel: Begriffe nennen und Sätze formulieren

Aufgabe: Wie heisst das in Österreich? Nennen Sie die Begriffe, die Sie schon in den obigen Aufgaben gelernt haben. Halten Sie sich dabei an die Standardaussprache, indem die österreichische Standardaussprache nicht ausgeschlossen werden kann. Formulieren Sie eigene Sätze.

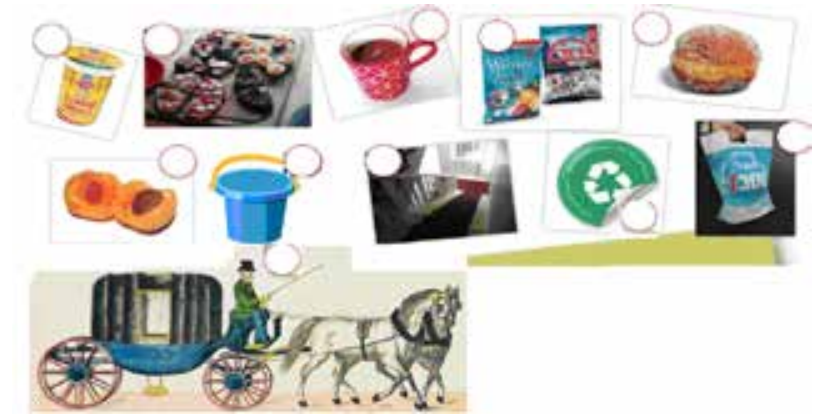

Nach der lexikalisch-phonetischen Arbeit wird den Lernenden ein Text zum Lesen und Hören angeboten. Als phonetische Vorübung kann die Aussprache des [1]-Lautes im Auslaut geklärt werden, damit man österreichische Merkmale der Aussprache beim Wahrnehmen nicht übergehen kann. Dazu demonstriert die Lehrkraft die Bilder mit den Datteln, Lebensmitteln und Mandeln. Dazu kommt die Erklärung, dass das auslautende [1] im Suffix -el nach Frikativen und Plosiven im Vergleich zur bundesdeutschen Standardaussprache nicht weich realisiert wird. [8, S.50]

$\mathrm{Zu}$ den phonetischen Eigenschaften, die für die Standardaussprache in Österreich verbindlich sind, gehört z.B. die Aussprache des [ $\underline{\dagger}]$-Lautes am Ende des Wortes im Suffix -el. Diese Aussprache kommt besonders im Raum Wien vor, man spricht ihn dunkel 
aus. Ausserdem fällt das Schwa [ə] in den Endungen aus. Nach den Velaren g, k und dem Nasal [ $\mathrm{\eta}$ ]; nach Frikativ, Plosiv und Affrikaten fällt das Schwa beim Sprechen aus. [5, S.73; 8. S.50]

Übung 7. Ziel: Die österreichischen Merkmale der Aussprache hören und die Informationen wahrnehmen und verstehen

Aufgabe: Sehen Sie sich eine österreichische Kochsendung "Köstlich kulinarisch mit „Süße Rohkost" unter der Internet-Adresse https://www.youtube.com/watch? $v=j a 13 A 4 b 7 i u c$ an. Was sagt B. Heider? Beachten Sie seine Realisierug des [ $\underline{\dagger}]$-Lautes am Ende des Wortes.

Die Lernenden sehen sich den Film noch einmal an und notieren sich das Rezept einer Torte. Dabei berücksichtigen sie die Aussprache des Konditors B. Heider und markieren die Wörter mit dem auslautenden -el, die er erwähnt. Dabei beachten sie die Realisierung des [1]-Lautes am Ende des Wortes. Folgende Wörter sind beachtenswert: Viertel, Lebensmittel, Mandel, ein bissel, Dattel.

Zum Schluss erhalten die Lernenden eine Liste der Bilder, die sie beschriften sollen. Sie versuchen die österreichische Aussprache zu imitieren, um die Sprachkultur des Landes zu identifizieren. Es ist eine kreative und produktiv-kommunikataive Aufgabe, wenn die Deutschlernenden fiktiv im österreichischen Supermarkt ein Gespräch mit einer Verkäuferin zu führen versuchen. Da gibt es zwei Personen. Eine Kundin sucht Lebensmittel heraus und eine Verkäuferin ist aufgrund ihrer Höflichkeit stets behilflich.

Übung 8. Ziel: ein Gespräch führen; österreichische Identität tolerieren

Aufgabe: Sie haben beschlossen, eine Kokostorte zuzubereiten. So sind Sie im österreichischen Supermarkt "Hofer" und suchen die passenden Zutaten. Dabei hilft Ihnen eine Verkäuferin aus Wien. Sie nennt alle Lebensmittel, die Sie für die Torte brauchen, und möchte Ihnen ein paar Bonbons anbieten.

Führen Sie das Gespräch.
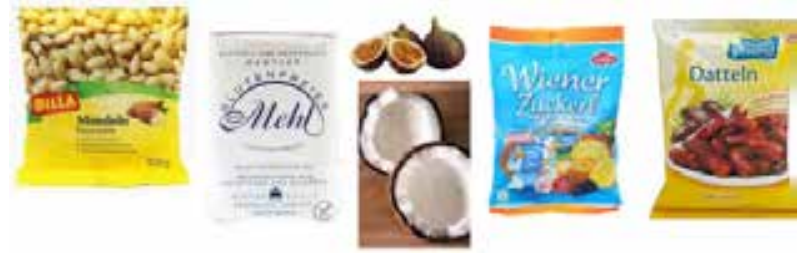

Mit einer solchen Aufgabe können die Lernenden das gelernte Vokabular im Alltag benutzen und im deutschsprachigen Land tolerant gegenüber neuen sprachlichen Erscheinungen sein.

Zusammenfassend lässt sich sagen, dass die Lehrkraft ohne Weiteres mithilfe einer Übungsreihe eine virtuelle Reise ins deutschsprachige Land Österreich mit seinen linguistischen Besonderheiten machen kann. Wichtig ist, dass die angebotenen Lernmaterialien den DaF-Lernenden ein Bewusstsein für die Differenziertheit des Deutschen geben.

Die Entwicklung der soziokulturellen Kompetenz ist kein selbstverständlicher Prozess. Das Vorhandensein der soziokulturellen Beziehungen im Leben eines Menschen, z.B. unter Kollegen oder Mitarbeitern, die aus einer anderen Kultur stammen und deren nationalen Merkmale sich (auch) durch die Sprache zeigen, macht die Lernenden nicht automatisch soziokulturell kompetent. Die günstige Entwicklung erfordert sowohl langfristige Arbeit und Bemühungen der Lernmaterialherstellenden als auch bewusste Auseinandersetzung der Lernenden mit dem Lernmaterial. Dabei ist die Verbindung von Theorie und Praxis sehr wichtig.

Unseres Erachtens soll das soziolinguistische Erlernen des Deutschen in der alltäglichen Unterrichtsplanung berücksichtigt werden und zum expliziten Unterrichtsprinzip werden. Man kann sagen, dass die Deutschlernenden normtolerant sind, wenn sie die Gleichwertigkeit und Standardsprachlichkeit des österreichischen und schweizerischen Deutsch kennen und den plurizentrischen Ansatz in die Tat umsetzen.

Man kann sicher sein, dass die Präsentation der Lernmaterialien in Aufgaben und Übungen, wo die soziokulturellen Besonderheiten der deutschsprachigen Länder unterstrichen sind, den DaF-Lernenden erleichtert, die verschiedenen Varietäten der deutschen Sprache kennen zu lernen oder über aktuelle Themen zu informieren.

Es sei betont: Ohne das Internet und die damit verbundenen Recherche- und Kommunikationsmöglichkeiten wäre die Bekanntschaft mit den soziokulturellen Besonderheiten des Deutschen hinsichtlich der nationalen Varietäten wohl kaum möglich. In erster Linie helfen den DaF-Lehrenden und -Lernenden die Internet-Seiten von ORF und SRF (österreichisches Fernsehen und schweizerisches Fernsehen), unterschiedliche Formen des authentischen Deutschen kennen zu lernen und $\mathrm{zu}$ erkennen, wo die österreichische und schweizerische deutsche Standardsprache benutzt wird.

Die vorgestellte Untersuchung und der gewählte Zugang zur Förderung der soziokulturellen Kompetenz hat Modellcharakter für die Entwicklung der fremdsprachlichen Kompetenzen auch in den anderen Fremdsprachen. 


\section{Literatur:}

1. Бориско Н.Ф. Curriculum für den sprachpraktischen Deutschunterricht an pädagogischen Fakultäten der Universitäten und pädagogischen Hochschulen. Kiew : Lenwit, 2004. 256 S.

2. Ammon U. Die Standardsprache in Deutschland, Österreich und der Schweiz. Das Problem der nationalen Varietäten. Gruyter Verlag. 1991. 575 S.

3. Czinglar Ch. Österreichische Varietäten in der DaF/DaZ-Lehrerausbildung. Universität Wien, 2009. 54 S.

4. Ebner J. Duden Österreichisches Deutsch Eine Einführung von Jakob Ebner. Dudenverlag Wien 2008. $52 \mathrm{~S}$.

5. Ehrlich K.Stimmbildung und Sprecherziehung. Ein Lehr-und Übungsbuch / Karoline Ehrlich. Bählau V erlag, Weimar. $2001336 \mathrm{~S}$.

6. Hirschfeld U. Phonetik im Kontext mündlicher Fertigkeiten / Ursula Hirschfeld. Babylonia № 2. 2011. S.10-17.

7. Kellermeier-Rehbein B. Plurizentrik Einführung in die Nationalen Varietäten des Deutschen / Birte Kellermeier-Rehbein Erich Schmidt Verlag, Berlin. 2014. 264 S.

8. Muhr R. Österreichisches Aussprachewörterbuch. Österreichische Aussprachedatenbank. 2007. Wien. $525 \mathrm{~S}$.

9. Reinke K. Phonetiktrainer B1 / Kerstin Reinke. Ernst Klett Sprachen Stuttgart. 2012. 104 S.

10. R. de Chillia Varietäten Deutsch. Deutsch als plurizentrische Sprache und DaF-Unterricht / in Begegnungssprache Deutsch - Motivation, Hearausforderung, Perspektiven / Rudlof de Chillia / StudienVerlag. 2006. Wien. - S. 51-65.

11. Takahashi H. Verschiedene Varietäten des Deutschen und deren Beziehung zum Unterricht Deutsch als Fremdsprachen (DaF) / Hideaki Takahashi. in: Zeitschrift für angewandte Linguistik. 2000. S. 19-33.

12. Trim J., North B. und Coste D. Gemeinsamer europäischer Referenzrahmen für Sprachen: lernen, lehren, beurteilen / John Trim, Brian North und Daniel Coste. Langenscheidt 2001. 244 S.

13. Takahashi H. Die richtige Aussprache des Deutschen in Deutschland, Österreich und der Schweiz. 1996. $300 \mathrm{~S}$.

14. Wiesinger P. Das österreichische Deutsch in Gegenwart und Geschichte / 3., aktual. und neuerl. erweit. Aufl., Wien. 2014. 428 S.

\section{Lehrwerke:}

1. Навчально-методичний комплекс DU 2; Підручник для студ.вищ.навч.закладів / Бориско Наталія, Каспер-Хене Хільтрауд, Бондаренко Ельвіра та ін. - Вінниця: Нова Книга, 2011. - 344 с.

2. DU 3. Навчально-методичний комплекс: підручник / Бориско Наталія, Каспер-Хене Хільтрауд, Васильченко Елена та інш. - Вінниця: Нова Книга, 2013. - 200 с.

3. Aspekte Mittelstufe Deutsch (B1+) Cover Ute Koithan, Nana Ochmann, Helen Schmitz, u. a. Langenscheidt KG, Berlin und München. - 2007. - S. 192

\section{Свиридюк В. П. ВИКОРИСТАННЯ АВСТРІАЦИЗМІВ ДЛЯ РОЗВИТКУ МОВНОЇ І МОВЛЕННСВОЇ КОМПЕТЕНТНОСТІ У СТУДЕНТІВ-ФІЛОЛОГІВ}

У статті йдеться про розвиток соиіокультурної компетентності через застосування австріацизмів на занятті з німецькоїмови, а також акиентується увага на плюріцентричності німецькоїмови як передумови формування лінгвосоиіокультурної компетентності володіння інщомовним спілкуванням унімецькомовному просторі. Особливу увагу зосереджено на комплексі вправ для ознайомлення з австрійським варіантом німецькоїмови на лексичному рівні. У статті звертається увага на формування фонетичних навичок і вмінь із метою сприйнятття та розуміння австрацизмів на заняттяях з німецької мови. Приділяється увага роботі над лексичними одиничями, австріацизмами на граматичному та фонетичному рівнях. Представлено опис фонетичних особливостей лексичних одиничь та описані їхні сегментарні ознаки в поточі мовлення. Наведені приклади вправ для демонстраиії використання австріацизмів у комунікативних ситуащіях для розвитку усного та писемного мовленні німещької мови. Стаття присвячена пошуку шляхів оптимізаціі розвитку навичок використання австріачизмів у письмі та говорінні студентів-філологів. Наведено опис інтерактивних онлайн-вправ для застосування у навчанні формування комунікативних навичок $i$ вмінь із німецькоїмови. У статті розкриваються приклади інтерактивних вправ для навчання формулювання речень німещькою мовою із застосуванням австріачизмів. У роботі підкреслюється важливість ознайомлення з національними варіантами німецької мови та розщирення тезаурусу студентів через використання австріацизмів і вдосконалення ними лінгвосочіокультурної компетентності.

Ключові слова: методи, розробка навчального матеріалу, начіональний варіант австрійської німецької мови, соціолінгвістична компетентність, фонетична компетентність, лексична компетентність. 